\title{
Effect of Temperature, Time and Diimide/Rubber Ratio on the Hydrogenation of Liquid Natural Rubber by Response Surface Methodology
}

\author{
Mohamad Shahrul Fizree Idris ${ }^{1}$, Nur Hanis Adila Azhar ${ }^{1}$, Fazira Firdaus ${ }^{1}$, \\ Siti Efliza Ashari ${ }^{2}$, and Siti Fairus Mohd Yusoff ${ }^{1,3, *}$
}

${ }^{1}$ School of Chemical Sciences and Food Technology, Faculty of Science and Technology, Universiti Kebangsaan Malaysia, 43600 Bangi, Selangor, Malaysia

${ }^{2}$ Department of Chemistry, Faculty of Science, Universiti Putra Malaysia, Jl. UPM, 43400 Serdang, Selangor, Malaysia

${ }^{3}$ Polymer Research Center (PORCE), Faculty of Science and Technology, Universiti Kebangsaan Malaysia, 43600 Bangi, Selangor, Malaysia

\section{* Corresponding author:}

email:sitifairus@ukm.edu.my

Received: July 4, 2018

Accepted: October 30, 2018

DOI: $10.22146 /$ ijc.36706

\begin{abstract}
Hydrogenated liquid natural rubber (HLNR) was synthesized from liquid natural rubber (LNR) by thermolysis of p-toluenesulfonyl hydrazide (TSH). The HLNR structure was characterized by Fourier-transform infrared (FTIR) and nuclear magnetic resonance (NMR) spectroscopies. Thermogravimetric analysis (TGA) showed that the HLNR had higher decomposition temperature compared to LNR. A response surface methodology (RSM) based on a central composite rotatable design (CCRD) with fivelevel-three-factors was used to optimize the main important reaction parameters, such as the TSH:LNR weight ratio (1-3), reaction temperature $\left(110-150{ }^{\circ} \mathrm{C}\right)$, and reaction time (1-8 h). A quadratic model was developed using this multivariate statistical analysis. Optimum conditions for the non-catalytic hydrogenation of LNR using TSH were obtained; an LNR hydrogenation percentage of $83.47 \%$ at a TSH:LNR weight ratio of 1.41 , a reaction temperature of $118.11^{\circ} \mathrm{C}$, and a reaction time of $3.84 \mathrm{~h}$ were predicted. The $R^{2}$ value of 0.9949 indicates that the model provides data that are well matched with those from the experiment.
\end{abstract}

Keywords: response surface methodology; central composite rotatable design; optimization; liquid natural rubber; hydrogenation

\section{- INTRODUCTION}

Rubbers are elastomers obtained from either natural or non-natural resources. Most natural rubbers (NRs) are synthesized by members of the Euphorbiaceae or Compositaceae families of plants, and by some fungi [1]. Generally, NR consists of isoprene units $\left(\mathrm{C}_{5} \mathrm{H}_{8}\right)$, each containing a single cis-configured double bond [2-3]. The natural polymer derived from the rubber tree, Hevea brasiliensis, is composed of cis-1,4-polyisoprene chains. This polymer is well known because of its elastic properties and high mechanical strength and has been used for a wide range of applications including transportation, agriculture, and insulation. However, NR has a low resistance to heat, weather, and chemical reagents. By increasing the level of saturation in the polymer, the stability of NR against thermal and oxidative degradation can be enhanced [4].

Depolymerization of the polyisoprene chains in NR can generate a more useful material that is referred to as "liquid natural rubber" (LNR). Generally, LNR has shorter polyisoprene chains and lower molecular weights $\left(M_{w}<10^{5}\right)$ than NR [4]. The Institut de Recherches sur le Caoutchouc has defined LNR to be a modified NR that can be pumped or poured without the assistance of any external medium as a solvent [5]. Nor and Ebdon also reported that LNR is able to flow at room temperature, thereby facilitating mixing and lower processing costs compared to NR [6]. LNR is considered 


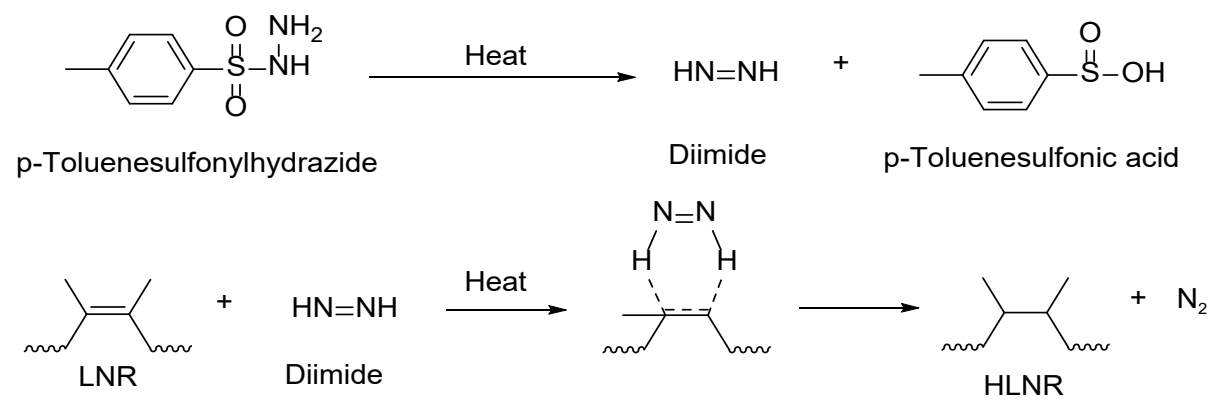

Scheme 1. Thermolysis of TSH and hydrogenation of unsaturated polymer by diimide

to be an important modified NR because the structure of the main polyisoprene chain is unaltered. LNR is mainly produced to lower the viscosity of NR so that the energy required for subsequent processes is reduced. LNR can be synthesized by thermal oxidation, photodegradation, redox chemistry, ozonolysis, and sonochemistry, after which it is easily processed to produce a variety of products [7]. LNR has two major advantages over synthetic rubbers. Firstly, the synthetic route for the preparation of LNR is greener, more renewable, and energy efficient compared to other technologies such as metathesis, degradation, or cleavage by periodic acid/lead tetraacetate [8-10]. Secondly, NR has a natural origin; it is synthesized by the $H$. brasiliensis tree.

Hydrogenation is a simple method for polymer modification and reduces the degree of unsaturation present in the polymer. Saturated moieties in the polymer lead to improved physical properties, such as stability against thermal, oxidative, and radiation-induced degradation [11-13]. The saturation of NR by hydrogenation can be carried out either by catalytic or non-catalytic methods. Catalytic hydrogenation is costly because it uses rare transition-metal catalysts. In addition, it often requires a special reactor such as an autoclave reactor or a Parr hydrogenator. Saturation by noncatalytic hydrogenation can easily be carried out using diimide that provides hydrogen atoms to the $\mathrm{C}=\mathrm{C}$ bonds (Scheme 1). In addition, non-catalytic hydrogenation uses less expensive reagents and milder conditions (e.g., lower pressures) compared to catalytic hydrogenation, and requires only simple apparatus [14-15]. Mahittikul et al. reported the hydrogenation of natural rubber latex (NRL) by thermolysis in the presence of $p$-toluenesulfonyl hydrazide (TSH) [16]. Recently, the hydrogenation of
LNR using diimide sources (TSH and 2,4,6trimethylbenzenesulfonyl hydrazide) has also been studied by Azhar et al. and Jamaluddin et al. [4,17].

Traditionally, the optimization of a reaction is carried out by varying one variable at a time. The major deficiency of this approach is that it does not reveal correlations among the studied variables. As a result, this technique does not reflect the full effects of each variable on the response [18]. This technique also leads to the high consumption of reagents and materials due to its single-factor optimization pathway. In order to overcome this problem, the optimization procedure needs to be carried out using the most relevant multivariate statistical technique, which is the response surface methodology (RSM). It is especially appropriate for analyzing and modeling multi-factor experiments because it can assess both single and interaction effects of specific factors [19]. The main advantage of RSM is the reduced number of experiments needed to provide sufficient information for a statistically acceptable result [20-21]. Azhar et al. and Jamaluddin et al. optimized the production of hydrogenated liquid natural rubber (HLNR) using conventional methods and concluded that the optimum TSH:LNR weight ratio is 3:1, leading to over $90 \%$ LNR hydrogenation after $4-6 \mathrm{~h}$ of reaction time at $130^{\circ} \mathrm{C}[4,17]$.

The objective of this work was to determine the optimum conditions for the production of HLNR by the thermolysis of LNR in the presence of TSH using RSM. The novelty of this work was the interactive effects of all parameters and the optimum conditions for the hydrogenation of LNR were determined statistically. The parameters used in this study were selected based on previous studies [17]. 
Table 1. Independent variables and their actual coded values

\begin{tabular}{lrrrrr}
\hline \multirow{2}{*}{ Variable } & \multicolumn{5}{c}{ Levels } \\
\cline { 2 - 6 } & \multicolumn{1}{c}{-2} & \multicolumn{1}{c}{0} & \multicolumn{1}{c}{+1} & \multicolumn{1}{c}{+2} \\
\hline TSH:LNR weight ratio, A & 1.00 & 1.41 & 2.00 & 2.59 & 3.00 \\
Reaction temperature, B $\left({ }^{\circ} \mathrm{C}\right)$ & 110.00 & 118.11 & 130.00 & 141.89 & 150.00 \\
Reaction time, C $(\mathrm{h})$ & 1.00 & 2.42 & 4.50 & 6.58 & 8.00 \\
\hline
\end{tabular}

\section{- EXPERIMENTAL SECTION}

\section{Materials}

In this work, natural rubber was supplied by the Rubber Research Institute of Malaysia (RRIM). Reagent grade toluene (> 99\%), methanol (> 99.8\%) and ethanol (95\%) were purchased from R\&M Chemicals (Himachal Pradesh). Reagent grade methylene blue ( $\geq 95 \%)$, rose bengal (95\%), and $p$-toluenesulfonyl hydrazide (TSH) (97\%) were purchased from Sigma Aldrich (Missouri).

\section{Instrumentation}

Fourier-transform infrared (FTIR) spectroscopy (Agilent Cary 630 FTIR) was employed to monitor any changes in the main LNR spectral bands upon hydrogenation. ${ }^{1} \mathrm{H}$ Nuclear Magnetic Resonance (NMR) spectroscopy was performed in $\mathrm{CDCl}_{3}$ at $400 \mathrm{MHz}$ on a Bruker AVANCE III HD instrument. The ${ }^{1} \mathrm{H}$ NMR signals were integrated in order to determine the hydrogenation percentage. The decomposition temperature of the products was determined using differential thermal analysis (DTA) (TGA/SDTA 851e, Mettler Toledo).

\section{Procedure}

\section{Preparation of $L N R$}

The LNR used in this study was prepared by the photosensitized degradation of NR as follows [22]. About $1 \mathrm{~kg}$ of natural rubber (NR) was soaked in toluene until it was completely swollen. Methanol $(10 \mathrm{~mL})$, rose bengal $(0.100 \mathrm{~g})$, and methylene blue $(0.066 \mathrm{~g})$ were added to the swollen NR, and the mixture was stirred with a mechanical stirrer at $10-30 \mathrm{rpm}$ for 12 days under visible light at room temperature. The product was then centrifuged at $6000 \mathrm{rpm}$ to separate the gel formed during photodegradation.

\section{Hydrogenation of LNR}

A solution of LNR with $10 \%$ dried rubber content was prepared [23]. Different amounts of TSH were added to fixed amounts of LNR (3.00 g) such that TSH:LNR ratios between 1:1 and 3:1 were obtained. The mixtures were stirred at $650 \mathrm{rpm}$ at different temperatures (110$\left.150{ }^{\circ} \mathrm{C}\right)$ and reaction times $(1-8 \mathrm{~h})$. The products were filtered and coagulated with ethanol to remove residual $\mathrm{TSH}$, after which they were dried in a vacuum oven to remove the remaining solvent.

\section{Experimental design and statistical analysis}

In this study, RSM with a five-level-three-factor CCRD was used, which required 20 experiments. The fractional factorial design was composed of eight factorial points, eight axial points, and six center points. Correlations between independent variables were determined by variance analysis (ANOVA). The sufficiency of the final model was determined from the differences between the experimental values and the predicted values. The proposed model was verified by validation experiments. Reactions were carried out with different reaction conditions, as described above. Table 1 lists the independent variables in terms of their actual coded values. The range of variables was selected on the basis of the studies of Jamaluddin et al. [17].

\section{- RESULTS AND DISCUSSION}

\section{Model Fitting and ANOVA}

An appropriate model with three parameter levels, including the TSH:LNR weight ratio, reaction temperature, and reaction time was selected for the optimization of HLNR via CCRD. The experimental and predicted hydrogenation percentages are listed in Table 2. Model-fitting was required to ensure that the predicted hydrogenation percentages correlated sufficiently with the experimental data. 
Table 2. CCRD of LNR hydrogenation percentages

\begin{tabular}{lccccc}
\hline Run & TSH:LNR weight ratio, & Reaction temperature, & Reaction time, & \multicolumn{2}{c}{ Hydrogenation $(\%)$} \\
\cline { 5 - 6 } no. & $\mathrm{A}$ & $\mathrm{B}\left({ }^{\circ} \mathrm{C}\right)$ & $\mathrm{C}(\mathrm{h})$ & Actual & Predicted \\
\hline 1 & $2.00(0)$ & $130.00(0)$ & $4.50(0)$ & 97.00 & 95.69 \\
2 & $2.00(0)$ & $130.00(0)$ & $8.00(+2)$ & 99.41 & 98.69 \\
3 & $2.00(0)$ & $130.00(0)$ & $4.50(0)$ & 96.24 & 95.69 \\
4 & $1.41(-1)$ & $118.11(-1)$ & $6.58(+1)$ & 95.61 & 95.10 \\
5 & $2.00(0)$ & $110.00(-2)$ & $4.50(0)$ & 90.09 & 90.74 \\
6 & $1.41(-1)$ & $141.89(+1)$ & $6.58(+1)$ & 96.93 & 97.39 \\
7 & $1.00(-2)$ & $130.00(0)$ & $4.50(0)$ & 81.26 & 82.20 \\
8 & $2.00(0)$ & $130.00(0)$ & $4.50(0)$ & 94.72 & 95.69 \\
9 & $1.41(-1)$ & $141.89(+1)$ & $2.42(-1)$ & 72.53 & 71.14 \\
10 & $1.41(-1)$ & $118.11(-1)$ & $2.42(-1)$ & 67.80 & 67.12 \\
11 & $2.59(+1)$ & $141.89(+1)$ & $2.42(-1)$ & 85.39 & 85.21 \\
12 & $2.59(+1)$ & $118.11(-1)$ & $6.58(+1)$ & 98.72 & 99.43 \\
13 & $2.00(0)$ & $130.00(0)$ & $4.50(0)$ & 95.55 & 95.69 \\
14 & $2.00(0)$ & $130.00(0)$ & $4.50(0)$ & 95.46 & 95.69 \\
15 & $2.00(0)$ & $150.00(+2)$ & $4.50(0)$ & 96.70 & 97.03 \\
17 & $3.00(+2)$ & $130.00(0)$ & $4.50(0)$ & 97.65 & 97.68 \\
18 & $2.00(0)$ & $130.00(0)$ & $1.00(-2)$ & 58.59 & 60.28 \\
19 & $2.00(0)$ & $130.00(0)$ & $4.50(0)$ & 95.35 & 95.69 \\
20 & $2.59(+1)$ & $118.11(-1)$ & $2.42(-1)$ & 81.16 & 80.01 \\
\hline
\end{tabular}

Table 3. ANOVA data for the quadratic model developed for hydrogenation of LNR

\begin{tabular}{lcccrr}
\hline Source & Sum of Squares & Degrees of Freedom & Mean Square & $F$-value & $P$-value \\
\hline Model & 2484.14 & 9 & 276.02 & 196.00 & $<0.0001$ \\
TSH:LNR weight ratio, A & 236.76 & 1 & 236.76 & 168.12 & $<0.0001$ \\
Reaction temperature, B & 39.18 & 1 & 39.18 & 27.82 & 0.0005 \\
Reaction time, C & 1457.11 & 1 & 1457.11 & 1034.71 & $<0.0001$ \\
AB & 0.51 & 1 & 0.51 & 0.36 & 0.5640 \\
AC & 26.59 & 1 & 26.59 & 18.88 & 0.0019 \\
BC & 1.09 & 1 & 1.09 & 0.78 & 0.4012 \\
$\mathrm{~A}^{2}$ & 57.85 & 1 & 57.85 & 41.08 & 0.0001 \\
$\mathrm{~B}^{2}$ & 5.70 & 1 & 5.70 & 4.05 & 0.0750 \\
$\mathrm{C}^{2}$ & 459.19 & 1 & 459.19 & 326.08 & $<0.0001$ \\
Residual & 12.67 & 9 & 1.41 & - & - \\
Lack-of-fit & 9.52 & 4 & 2.38 & 3.78 & 0.0887 \\
Pure error & 3.15 & 5 & 0.63 & - & - \\
Corrected total & 2496.81 & 18 & - & - & - \\
\hline
\end{tabular}

The experimental data were fitted to several models including a linear model, a two-factorial-interaction model, a quadratic model, and a cubic model; ANOVA revealed that the hydrogenation of liquid natural rubber is most suitably described by a quadratic polynomial model. The fitted quadratic polynomial is expressed as:
Hydrogenation $(\%)=+95.69+4.60 \mathrm{~A}+1.87 \mathrm{~B}$

$$
\begin{aligned}
& +11.42 \mathrm{C}+0.30 \mathrm{AB}-2.14 \mathrm{AC} \\
& -0.43 \mathrm{BC}-2.03 \mathrm{~A}^{2}-0.64 \mathrm{~B}^{2}-5.73 \mathrm{C}^{2}
\end{aligned}
$$

where $\mathrm{A}$ is the TSH:LNR weight ratio, $\mathrm{B}$ is the reaction temperature, and $\mathrm{C}$ is the reaction time. A positive sign indicates a synergistic effect, whereas a negative sign 
indicates an antagonistic effect. A negative coefficient indicates that the associated parameter has a negative influence on the percentage of hydrogenation. This quadratic model was found to have a coefficient of determination $\left(\mathrm{R}^{2}\right)$ of 0.9949 , which means that $99.49 \%$ of the total variation in the observed findings is attributable to the independent variables. Normally, a regression model with an $\mathrm{R}^{2}$ value above 0.9 is considered to be highly correlated [24]. Furthermore, previous studies found that empirical models better fit the actual data when $\mathrm{R}^{2}$ is close to unity [25]. Therefore, the high value of $\mathrm{R}^{2}$ obtained in our regression model indicates excellent agreement between the predicted and actual percentages of HLNR hydrogenation.

The ANOVA data for the model are tabulated in Table 3. The computed F-value of the model (196.00) indicates that the model is significant, while the lack-offit $F$-value of 3.78 shows that lack-of-fit is not significant relative to pure error. The model $F$-value had an associated $P$-value $(<0.0001)$ less than 0.05 , indicating and there is only a $0.01 \%$ chance that a model $F$-value this large could have occurred due to noise present in the experiments. A model is considered to be well-fitted to the

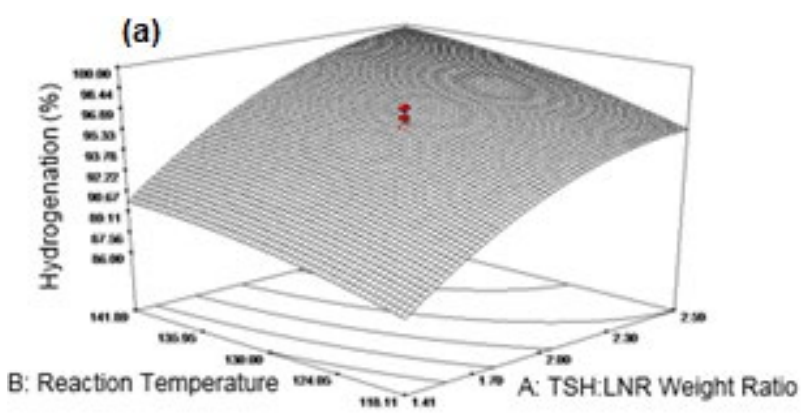

experimental data if it displays significant regression and a non-significant lack-of-fit [26]. A, B, and $C$ are the main linear terms, while $\mathrm{AB}, \mathrm{AC}$, and $\mathrm{BC}$ are the interaction terms, and $\mathrm{A}^{2}, \mathrm{~B}^{2}$, and $\mathrm{C}^{2}$ are the quadratic response terms. In this study, $\mathrm{A}, \mathrm{B}$, and $\mathrm{C}$ are significant $(\mathrm{p}<0.05)$, the interaction term $\mathrm{AC}$ is significant $(\mathrm{p}<$ $0.05)$, while quadratic terms $\mathrm{A}^{2}$ and $\mathrm{C}^{2}$ are also significant $(\mathrm{p}<0.05)$. Fig. 1 displays the good relationship between the actual and predicted LNR hydrogenation percentages. The linear distribution is indicative of a well-fitted model. The generated model

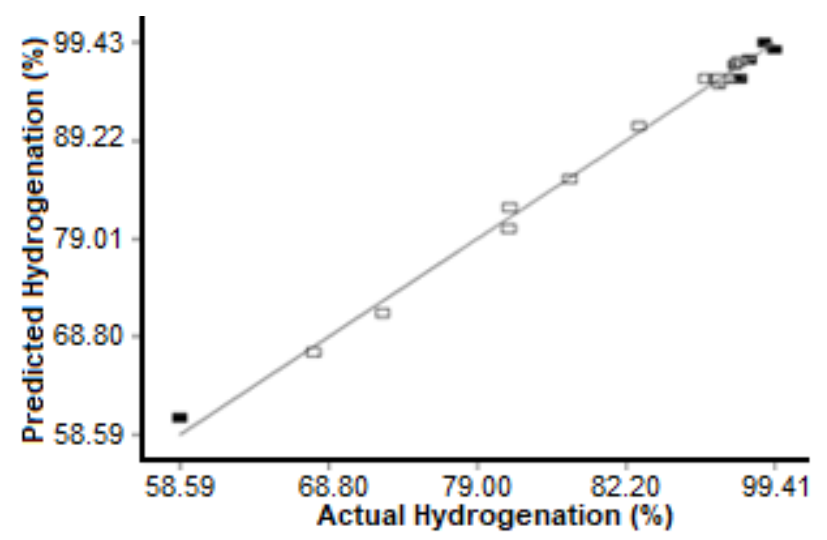

Fig 1. Correlation of actual and predicted value of hydrogenation of LNR

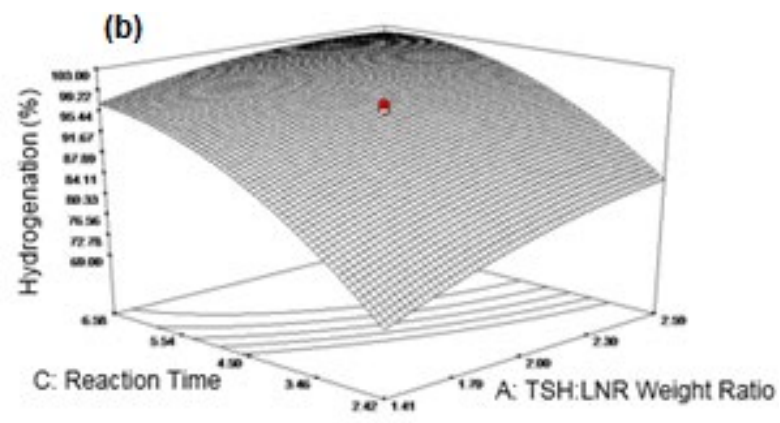

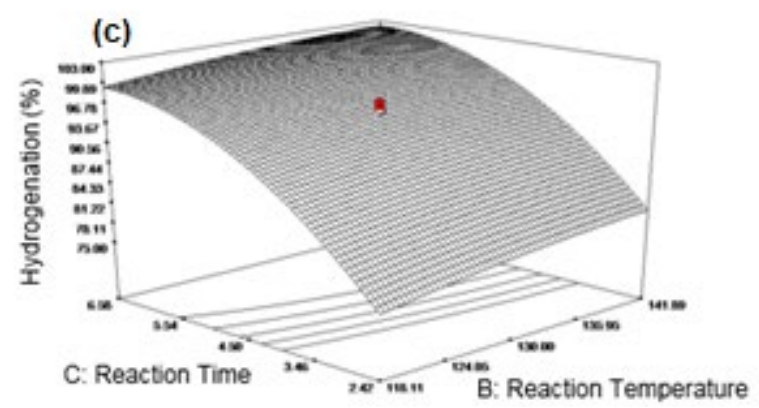

Fig 2. Three-dimensional response surface plots. (a) The weight ratio of TSH:LNR versus reaction time (h), (b) The weight ratio of TSH:LNR versus reaction temperature $\left({ }^{\circ} \mathrm{C}\right)$, and $(\mathrm{c})$ reaction time $(\mathrm{h})$ versus reaction temperature $\left({ }^{\circ} \mathrm{C}\right)$ on the percentage of hydrogenation as a response 
was subsequently employed to study the effect of various parameters and their interactions on the LNR hydrogenation percentage.

\section{Effect of Parameters}

The correspondence among all variables and response can be known by reviewing the threedimensional (3D) response surface plots. Fig. 2(a) displays the response surface as functions of the TSH:LNR weight ratio and reaction temperature over the 1.41-2.59 and $118.11-141.80{ }^{\circ} \mathrm{C}$ ranges, respectively, with the reaction time fixed at $4.50 \mathrm{~h}$. The percentage of hydrogenation increases with increasing reaction temperature; a reaction temperature of $141.89{ }^{\circ} \mathrm{C}$ and TSH:LNR weight ratio of 2.59 led to the maximum hydrogenation percentage (over 98\%). Based on the response surface plot (Fig. 2(a)), increasing the TSH:LNR weight ratio increases the hydrogenation percentage while increasing the reaction temperature has little effect. We conclude that the reaction temperature has a smaller effect on the LNR hydrogenation percentage compared to the TSH:LNR weight ratio. Jamaluddin et al. reported that $\mathrm{TSH}$ decomposes at about $100{ }^{\circ} \mathrm{C}$ [17]. Therefore, increases in reaction temperature contribute less to increases in hydrogenation percentage.

Fig. 2(b) illustrates the effect of varying the TSH:LNR weight ratio and reaction time over the 1.412.59 and 2.42-6.58 $\mathrm{h}$ ranges, while the reaction temperature was fixed at $130{ }^{\circ} \mathrm{C}$, and reveals that the maximum hydrogenation percentage (over 98\%) was obtained at a TSH:LNR weight ratio of 2.30 and at $6.50 \mathrm{~h}$. Hydrogenation cannot occur if the diimide is not generated by the thermolysis of TSH. In addition, longer reaction times lead to higher hydrogenation percentages as long as the diimide source remains in the reaction mixture. Once the TSH has been completely decomposed by thermolysis, hydrogenation cannot continue due to diimide deficiency. The TSH:LNR weight ratio also plays an important role in the hydrogenation of LNR. Azhar et al. and Jamaluddin et al. demonstrated optimum TSH:LNR weight ratios of 3:1 [4,17]. The conversion of the unsaturated bonds in LNR requires the optimum amount of TSH [4,17], and this amount depends considerably on the total weight of the LNR. The HLNR hydrogenation percentage produced remains fixed, even with prolonged reaction times, once all of the unsaturated bonds in LNR have reacted.

Fig. 2(c) shows the response surface as functions of reaction temperature $\left(118.11-141.89^{\circ} \mathrm{C}\right)$ and reaction time (2.42-6.58 h) at a fixed TSH:LNR weight ratio (2.0). The highest LNR hydrogenation percentage was obtained at $141.89{ }^{\circ} \mathrm{C}$ and $6.58 \mathrm{~h}$ of reaction time, whereas the lowest HLNR yield was obtained at 118.11 ${ }^{\circ} \mathrm{C}$ and $2.42 \mathrm{~h}$. As mentioned previously, the reaction temperature has a smaller effect on the hydrogenation percentage because the reaction temperature is above $100{ }^{\circ} \mathrm{C}$ (the temperature for TSH decomposition). From the surface plot, the hydrogenation percentage is observed to increase with increasing reaction time. This is because the conversion of the unsaturated bonds in LNR is mostly dependent to reaction time.

\section{Optimum Condition}

The optimum conditions for the hydrogenation of LNR were predicted using the optimization function of the Design Expert Software, which reveals the most desirable (optimum) conditions for the hydrogenation of LNR, as shown in Table 4. The highest LNR hydrogenation percentage is predicted to be $83.47 \%$. The optimum reaction parameters are $3.84 \mathrm{~h}$, a TSH:LNR weight ratio of 1.41 , and a reaction temperature of $118.11{ }^{\circ} \mathrm{C}$. The response surface can determine the optimal combination of parameters that provides the highest percentage under minimal reaction conditions; all parameters were assigned minimum values that achieved the maximum LNR hydrogenation percentage.

Table 4. Optimum conditions for the hydrogenation of LNR

\begin{tabular}{lccccccr}
\hline Run & TSH:LNR weight ratio, & Reaction temperature, & Reaction time, & \multicolumn{3}{c}{ Hydrogenation $(\%)$} & \multirow{2}{*}{ Deviation } \\
\cline { 5 - 6 } no. & $\mathrm{A}$ & $\mathrm{B}\left({ }^{\circ} \mathrm{C}\right)$ & $\mathrm{C}(\mathrm{h})$ & Actual & Predicted & \\
\hline 1 & 1.41 & 118.11 & 3.84 & 83.47 & 81.83 & 1.64 \\
\hline
\end{tabular}


Table 5. Validation of the quadratic model

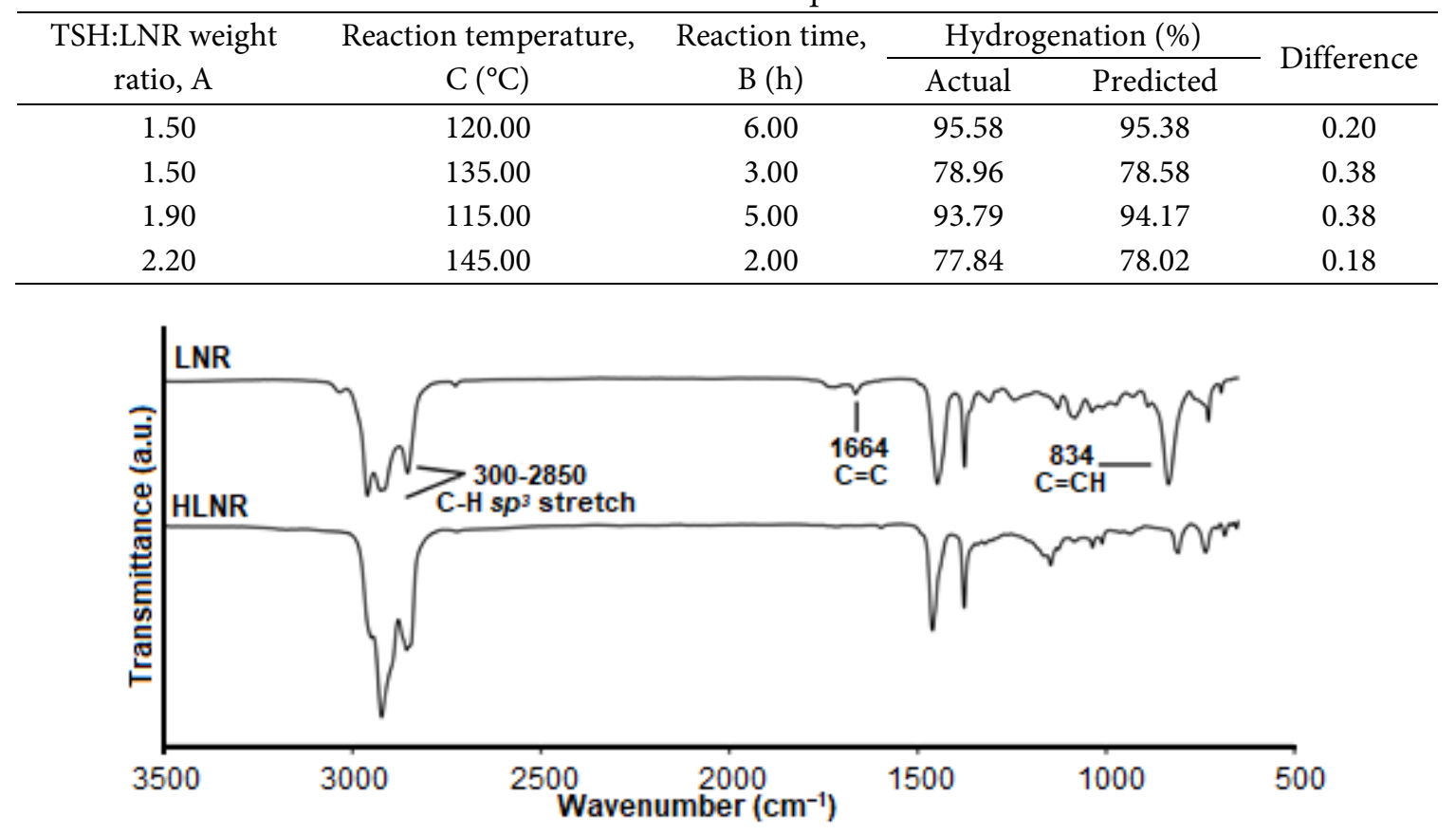

Fig 3. FTIR spectra of LNR and HLNR (99.41\%)

The quadratic model for the optimization of LNR hydrogenation was also validated, the results of which are listed in Table 5. Inspection of Table 5 reveals that different reaction conditions provide hydrogenation percentages that are still acceptable. A comparison of the predicted and actual data reveals a good correspondence between them, indicating that the empirical model derived by RSM can be used to adequately describe the relationship between the variables and their impact on the LNR hydrogenation percentage.

\section{Comparison of Parameter Study Techniques}

Based on the study conducted by Azhar et al., HLNR with hydrogenation percentage exceeding 90\% was obtained at TSH:LNR weight ratio of $3: 1$, a reaction temperature of $130{ }^{\circ} \mathrm{C}$ and reaction time of $4 \mathrm{~h}$. Then, Jamaluddin et al. stated that the ratio of TSH:LNR at 3:1 would produce HLNR with hydrogenation percentage above $90 \%$ after $6 \mathrm{~h}$ of reaction at $130^{\circ} \mathrm{C}$. In this study, the optimum condition of the hydrogenation reaction has been determined by setting all parameters in the minimum state for the maximum hydrogenation percentage. Such a situation cannot be done if it is carried out conventionally.
Additionally, the RSM method is also capable of providing $3 \mathrm{D}$ empirical models that allow researchers to understand the effect of each response parameter in depth.

\section{Identification of LNR and HLNR}

The product was characterized by FTIR and NMR spectroscopies. The formation of HLNR was confirmed by comparing its FTIR spectrum with that of LNR. The spectra of LNR and 99.41\%-hydrogenated HLNR are displayed in Fig. 3 and show characteristic absorption bands at 3000-2850, 1664, and $834 \mathrm{~cm}^{-1}$, which correspond to $s p^{3} \mathrm{C}-\mathrm{H}$ stretching, $\mathrm{C}=\mathrm{C}$ stretching, and olefinic $\mathrm{C}-\mathrm{H}$ bending, respectively. The intensity of the $s p^{3} \mathrm{C}-\mathrm{H}$ stretching band $\left(3000-2850 \mathrm{~cm}^{-1}\right)$ increases as a result of LNR hydrogenation, while the intensities of the other two peaks decrease due to the reduced levels of $\mathrm{C}=\mathrm{C}\left(1664 \mathrm{~cm}^{-1}\right)$ and olefinic $\mathrm{C}-\mathrm{H}\left(834 \mathrm{~cm}^{-1}\right)$ groups. Several bands between 1500 and $900 \mathrm{~cm}^{-1}$ in the spectrum of HLNR are more intense than those in the spectrum of LNR. The stronger signals in this region originate from paraffinic $\mathrm{C}-\mathrm{H}$ bending vibrations owing to the increased number of alkane groups following hydrogenation. 

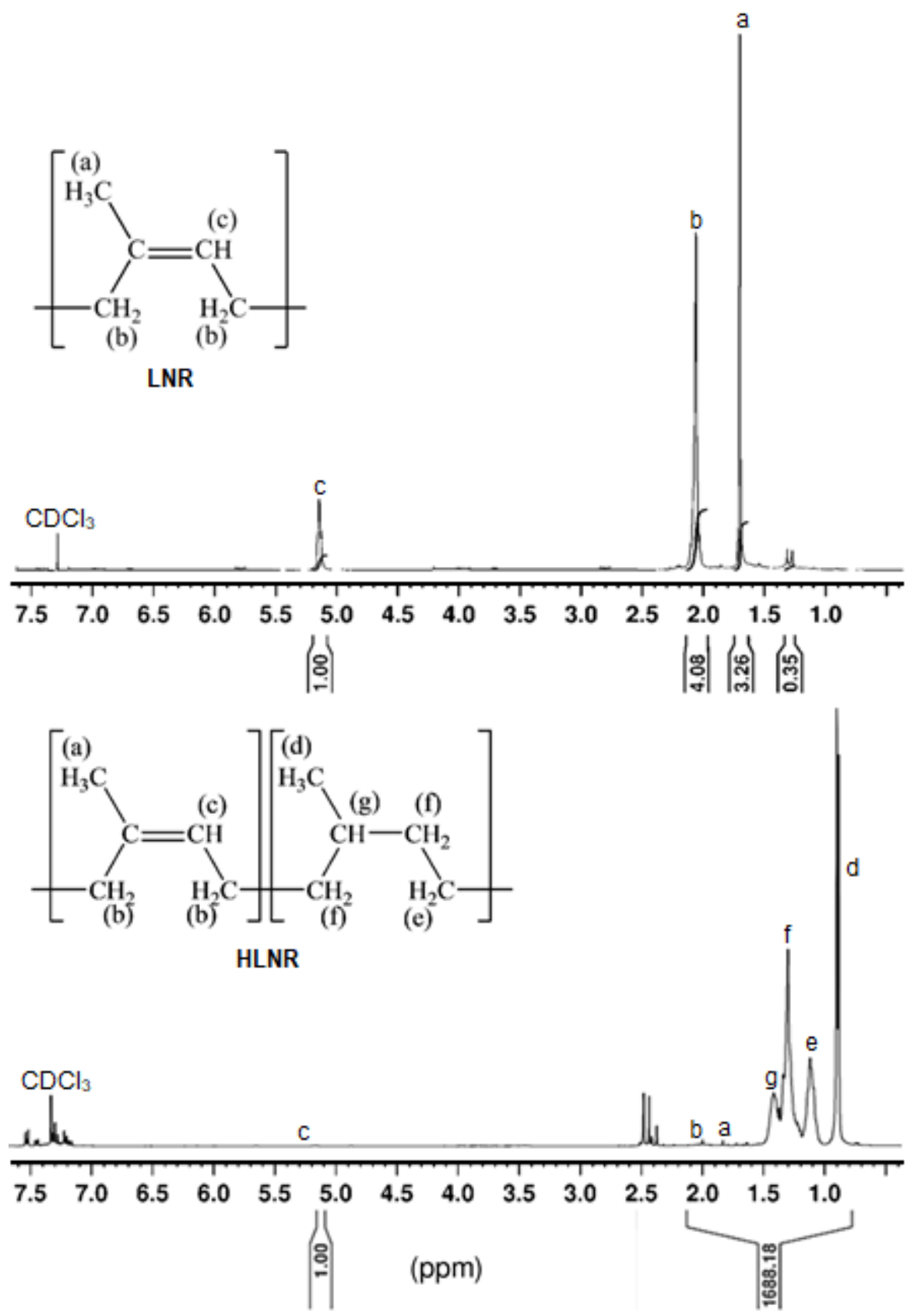

\%

Fig 4. ${ }^{1} \mathrm{H}$ NMR spectra of LNR and HLNR (99.41\%)

The ${ }^{1} \mathrm{H}$ NMR spectra of LNR and HLNR are displayed in Fig. 4. The spectrum of LNR displays signals for allylic methyl $\left(\mathrm{R}-\mathrm{CH}_{3}\right)$ and methylene $\left(\mathrm{R}-\mathrm{CH}_{2}-\mathrm{R}\right)$ protons, and olefinic $(\mathrm{R}-\mathrm{CH}=\mathrm{C}-\mathrm{R} 2)$ protons at $1.73,2.02$, and $5.12 \mathrm{ppm}$, respectively. These three signals are reduced in intensity in the spectrum of HLNR, and new signals appear in the $0.85-1.39 \mathrm{ppm}$ region that corresponds to the saturated hydrocarbon groups (methylene $\left(-\mathrm{CH}_{2}-\right)$, and methyl $\left(-\mathrm{CH}_{3}\right)$ ). The NMR signals of 99.41\% HLNR were broader than those of LNR because the rate of spin-lattice relaxation increases with increasing numbers of alkane groups upon hydrogenation. The ratio between the integrated olefinic proton signal and the signals in the range of $0.8-2.1 \mathrm{ppm}$ was used to calculate hydrogenation percentages [17]. The signals in the 2.3-2.5 ppm range correspond to benzylic protons from toluene and TSH. In conclusion, the FTIR and NMR spectra show that microstructural 


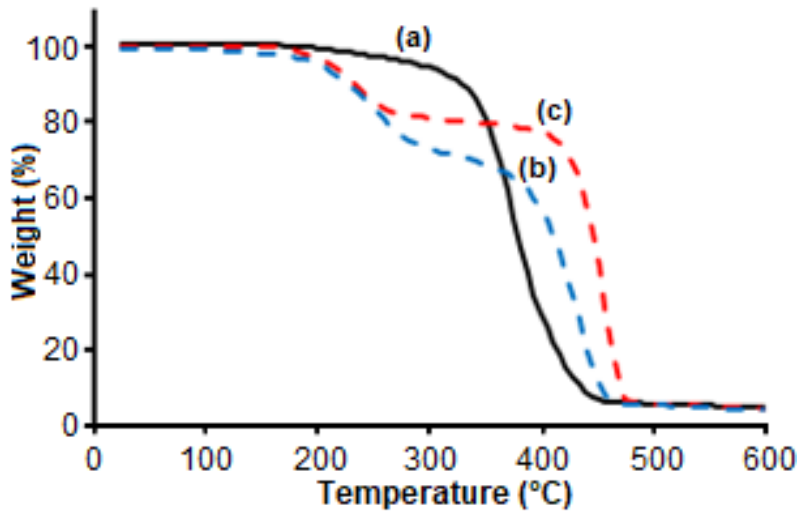

Fig 5. Thermograms of (a) LNR; (b) HLNR at $58.59 \%$ and (c) HLNR at $99.41 \%$

changes had occurred during the hydrogenation process.

\section{Thermal Behavior of LNR and HLNR}

Thermogravimetric analysis (TGA) was carried out to investigate the thermal properties of LNR and HLNR. Fig. 5 shows the thermograms of LNR and HLNR based on decomposition temperature. Thermogram of LNR shows a decomposition temperature at $380{ }^{\circ} \mathrm{C}$. However, thermogram of HLNR shows a two-step decomposition. The first stage of the decomposition is due to the side product of TSH decomposition at around $245^{\circ} \mathrm{C}$. It shows an increment in decomposition temperature of HLNR upon hydrogenation. Thermograms of HLNR at 58.59 and $99.41 \%$ hydrogenation show maximum decomposition temperature at 427 and $451{ }^{\circ} \mathrm{C}$, respectively. Based on the comparison between both HLNR thermograms, a higher percentage of conversion of HLNR also increased the decomposition temperature.

\section{- CONCLUSION}

The conditions for the formation of hydrogenated liquid natural rubber from liquid natural rubber were successfully optimized using response surface methodology (RSM) instead of conventional methods. The high coefficient of determination $\left(\mathrm{R}^{2}=0.9949\right)$ indicates that the predicted and experimental values are well correlated. ANOVA reveals that the model effectively describes the relationship between the liquid natural rubber hydrogenation percentage and three factors, the TSH:LNR weight ratio, the reaction temperature, and the reaction time. This model can be used to predict the percentage of LNR hydrogenation under any given set of conditions within the experimental range. The optimum conditions determined in this manner can be used to scale-up the process, minimize costs, and reduce reaction times. We have demonstrated that the optimum LNR hydrogenation percentage can be successfully predicted by RSM.

\section{- ACKNOWLEDGMENTS}

The authors acknowledge the Ministry of Higher Education (MOHE) and Universiti Kebangsaan Malaysia (UKM) for the research grants (FRGS/1/2016/STG01/UKM/02/4 and GUP-2017-004).

\section{- REFERENCES}

[1] Bode, H.B., Kerkhoff, K., and Jendrossek, D., 2001, Bacterial degradation of natural and synthetic rubber, Biomacromolecules, 2 (1), 295-303.

[2] Tanaka, Y., and Sakdapipanich, J.T., 2005, “Chemical Structure and Occurrence of Natural Polyisoprenes" in Biopolymers Online, Eds., Steinbüchel, A., Wiley$\mathrm{VCH}$ Verlag GmbH \& Co. KGaA.

[3] Rose, K., and Steinbüchel, A., 2005, Biodegradation of natural rubber and related compounds: Recent insights into a hardly understood catabolic capability of microorganisms, Appl. Environ. Microbiol., 71 (6), 2803-2812.

[4] Azhar, N.H.A., Jamaluddin, N., Md Rasid, H., Yusof, M.J.M., and Yusoff, S.F.M., 2015, Studies on hydrogenation of liquid natural rubber using diimide, Int. J. Polym. Sci., 2015, 243038.

[5] Institut de Recherches sur le Caouthchouc (IRCA), 1985, Development of liquid rubber, Final report, UNIDO, UF/GLO/81/059.

[6] Nor, H.M., and Ebdon, J.R., 1998, Telechelic liquid natural rubber: A review, Prog. Polym. Sci., 23 (2), 143-177.

[7] Tanaka, Y., Sakaki, T., Kawasaki, A., Hayashi, M., Kanamaru, E., and Shibata, K., 1999, Production process of depolymerized natural rubber, US Patents, US5856600 A. 
[8] Burfield, D., and Gan, S.N., 1977, Determination of epoxy groups in natural rubber by degradation methods, Polymer, 18 (6), 607-611.

[9] Gillier-Ritoit, S., Reyx, D., Campistron, I., Laguerre, A., and Singh, R.P., 2003, Telechelic cis-1,4oligoisoprenes through the selective oxidolysis of epoxidized monomer units and polyisoprenic monomer units in cis-1,4-polyisoprenes, J. Appl. Polym. Sci., 87 (1), 42-46.

[10] Thanki, P.N., Reyx, D., Campistron, I., Laguerre, A., and Singh, R.P., 2004, Metathetic alkenolysis of unsaturated units in polymers and copolymersapplication to the synthesis of epoxy-functionalized oligomers and organic compounds, Eur. Polym. J., 40 (11), 2611-2616.

[11] Rao, P.V.C., Upadhyay, V.K., and Pillai, S.M., 2001, Hydrogenation of polybutadienes catalyzed by $\mathrm{RuCl}_{2}\left(\mathrm{PPh}_{3}\right)_{3}$ and a structural study, Eur. Polym. J., 37 (6), 1159-1164.

[12] Singha, N.K., De, P.P., and Sivaram, S., 1997, Homogeneous catalytic hydrogenation of natural rubber using $\mathrm{RhCl}\left(\mathrm{PPh}_{3}\right)_{3}$, J. Appl. Polym. Sci., 66 (9), 1647-1652.

[13] Gan, S.N., Subramaniam, N., and Yahya, R., 1996, Hydrogenation of natural rubber using nickel 2-ethyl hexanoate catalyst in combination with triisobutyl aluminum, J. Appl. Polym. Sci., 59 (1), 63-70.

[14] Harwood, H.J., Russell, D.B., Verthe, J.J., and Zymonas, J., 1973, Diimide as a reagent for the hydrogenation of unsaturated polymers, Macromol. Chem. Phys., 163 (1), 1-12.

[15] Mango, L.A., and Lenz, R.W., 1973, Hydrogenation of unsaturated polymers with diimide, Macromol. Chem. Phys., 163 (1), 13-36.

[16] Mahittikul, A., Prasassarakich, P., and Rempel, G.L., 2007, Noncatalytic hydrogenation of natural rubber latex, J. Appl. Polym. Sci., 103 (5), 2885-2895.

[17] Jamaluddin, N., Yusof, M.J.M., Abdullah, I., and Yusoff, S.F.M., 2016, Synthesis, characterization, and properties of hydrogenated liquid natural rubber, Rubber Chem. Technol., 89 (2), 227-239.
[18] Lundstedt, T., Seifert, E., Abramo, L., Thelin, B., Nyström, A., Pettersen, J., and Bergman, R., 1998, Experimental design and optimization, Chemom. Intell. Lab. Syst., 42 (1), 3-40.

[19] Li, B., Wang, W., Wang, K., Zhang, D., Guan, L., and Liu, F., 2015, Thiacloprid suspension formula optimization by a response surface methodology, RSC Adv., 5 (34), 26654-26661.

[20] Gunawan, E.R., Basri, M., Rahman, M.B.A., Salleh, A.B., and Rahman, R.N.Z.A., 2005, Study on response surface methodology (RSM) of lipasecatalyzed synthesis of palm-based wax ester, Enzyme Microb. Technol., 37 (7), 739-744.

[21] Gunawan, E.R., and Suhendra, D., 2010, Fourfactor response surface optimization of the enzymatic synthesis of wax ester from palm kernel oil, Indones. J. Chem., 8 (1), 83-90.

[22] Abdullah, I., 1996, Process for manufacturing liquid natural rubber (LNR), Malaysian Patent, MY108852-A.

[23] Kargarzadeh, H., Ahmad, I., Abdullah, I., Thomas, R., Dufresne, A., Thomas, S., and Hassan, A., 2015, Functionalized liquid natural rubber and liquid epoxidized natural rubber: A promising green toughening agent for polyester, J. Appl. Polym. Sci., 132 (3), 41292.

[24] Li, Y., Lu, J., Gu, G., and Mao, Z., 2005, Characterization of the enzymatic degradation of arabinoxylans in grist containing wheat malt using response surface methodology, J. Am. Soc. Brew. Chem., 63 (4), 171-176.

[25] Hamzaoui, A.H., Jamoussi, B., and M'nif, A., 2008, Lithium recovery from highly concentrated solutions: Response surface methodology (RSM) process parameters optimization, Hydrometallurgy, 90 (1), 1-7.

[26] Bezerra, M.A., Santelli, R.E., Oliveira, E.P., Villar, L.S., and Escaleira, L.A., 2008, Response surface methodology (RSM) as a tool for optimization in analytical chemistry, Talanta, 76 (5), 965-977. 